\title{
Acute effects of insulin on skeletal muscle growth and differentiation genes in men with type 2 diabetes
}

\author{
Sandeep Dhindsa ${ }^{1,2, *}$, Husam Ghanim ${ }^{1, *}$, Kelly Green ${ }^{1}$, Sanaa Abuaysheh${ }^{1}$, Manav Batra', Antoine Makdissi', \\ Ajay Chaudhuri', Sartaj Sandhu' ${ }^{1}$ and Paresh Dandona ${ }^{1}$ \\ ${ }^{1}$ Division of Endocrinology, Diabetes and Metabolism, State University of New York at Buffalo, Williamsville, New \\ York, USA and 2Division of Endocrinology, Diabetes and Metabolism, Saint Louis University, St Louis, Missouri, USA \\ Correspondence \\ should be addressed \\ to P Dandona \\ *(S Dhindsa and H Ghanim contributed equally to this work) \\ Email \\ pdandona@KaleidaHealth.org
}

\begin{abstract}
Aims: Insulin has anabolic effects on skeletal muscle. However, there is limited understanding of the molecular mechanisms underlying this effect in humans. We evaluated whether the skeletal muscle expression of satellite cell activator fibroblast growth factor 2 (FGF2) and muscle growth and differentiation factors are modulated acutely by insulin during euglycemic-hyperinsulinemic clamp (EHC).

Design and methods: This is a secondary investigation and analysis of samples obtained from a previously completed trial investigating the effect of testosterone replacement in males with hypogonadotropic hypogonadism and type 2 diabetes. Twenty men with type 2 diabetes underwent quadriceps muscle biopsies before and after $4 \mathrm{~h}$ of EHC. Results: The infusion of insulin during EHC raised the expression of myogenic growth factors, myogenin (by $72 \pm 20 \%$ ) and myogenin differentiation protein (MyoD; by $81 \pm 22 \%$ ). Insulin reduced the expression of muscle hypertrophy suppressor, myogenic regulatory factor 4 (MRF4) by $34 \pm 14 \%$. In addition, there was an increase in expression of FGF receptor 2, but not FGF2, following EHC. The expression of myostatin did not change.

Conclusions: Insulin has an acute potent effect on expression of genes that can stimulate muscle differentiation and growth.
\end{abstract}

\section{Introduction}

Insulin is a major anabolic hormone and is known to support muscle growth (1). Insulin treatment in patients with uncontrolled diabetes increases muscle mass, while insulin deficiency leads to muscle loss. Patients with type 2 diabetes are known to have a relative deficiency of muscle mass and quality, presumably due to insulin resistance (2, $3)$. Insulin infusion in humans increases muscle protein synthesis and decreases muscle breakdown (4). However, the effect of insulin on genes that control skeletal muscle growth and differentiation in humans has not been studied, in vivo.

The initial step in muscle growth is recruitment of quiescent satellite cells. Fibroblast growth factor
2 (FGF2) has recently been shown to play a cardinal role in mice in the proliferation and development of satellite cells or muscle stem cells which mature into skeletal muscle cells $(5,6)$. Since nuclei in myofibers are terminally differentiated, muscle hypertrophy also largely involves activation of satellite cells (7, 8, 9). Further muscle development is dependent upon 4 myogenic regulatory factors: myogenic factor 5 (MYF5), myogenic differentiation protein (MyoD), myogenin and myogenic regulatory factor 4 (MRF4) (5). MYF5 is the first of these factors to be expressed in activated satellite cells. MyoD is the key transcription factor for myogenic differentiation, while myogenin is the mediator of

Published by Bioscientifica Ltd. 
myotube formation and myoblast fusion. MRF4, the most prominent regulatory factor expressed in mature muscle, is a suppressor of muscle hypertrophy $(10,11)$.

We have recently demonstrated a stimulatory action of testosterone on the plasma concentration of FGF2 and the expression of FGF2 in the skeletal muscle in patients with hypogonadotropic hypogonadism (HH) and type 2 diabetes (12). In addition, we found a suppressive effect of testosterone on the skeletal muscle expression of two negative muscle growth regulators: myostatin and MRF4 (12). This is relevant since testosterone has been shown to be an insulin sensitizer and stimulator of the classical insulin signal transduction pathway at four distinct levels by increasing the expression of IR $\beta$, IRS- 1, AKT2 and GLUT4 (13). In order to establish the effect of testosterone on insulin sensitivity, we carried out euglycemichyperinsulinemic clamps (EHC) in patients with $\mathrm{HH}$ and type 2 diabetes. This provided us with an opportunity to investigate the action of insulin on plasma concentrations of mediators of muscle growth and their expression in the skeletal muscle. Specifically, we evaluated the effect of EHC upon expression in skeletal muscle of (1) muscle growth factors: MyoD, myogenin and MYF5, (2) negative regulators of muscle growth: myostatin and MRF4, and (3) FGF2 and its receptors, FGFR1 and FGFR2. Effect of EHC on serum concentrations of FGF2 and myostatin was also evaluated.

\section{Methods}

The results presented in this paper are secondary analyses of a previously concluded study that was designed to evaluate the metabolic aspects of testosterone deficiency and its replacement in men with type 2 diabetes. The details on study design as well as the results on insulin sensitivity, inflammation and body composition from that trial have previously been published (13). Briefly, 50 eugonadal men and 44 hypogonadal men participated in the primary trial. All men had type 2 diabetes and were between the ages of 30 and 65 years. Their HbA1c was $\leq 8 \%$ and they had been on stable diabetes regimen for at least 3 months. The protocol was approved by the Human Research Board of the State University of New York at Buffalo, informed consent was signed by all subjects and the trial was registered with clinicaltrials.gov (NCT01127659) (13).

As part of study protocol, subjects were asked to undergo EHC, DEXA scans for lean body mass and fat mass measurements, and muscle biopsies. Out of 50 eugonadal men, only 20 men agreed to provide muscle biopsies both before and after EHC. We herein report the results obtained from muscle and serum samples from those 20 men.

\section{Hyperinsulinemic-euglycemic clamp (EHC)}

All subjects underwent EHC for $4 \mathrm{~h}$ (13). Oral hypoglycemic medications were held on the morning of EHC. Subjects on long-acting insulin were asked not to take their insulin the night before the clamp. Insulin clamp was started with a priming dose of short-acting human insulin (Humulin R; Eli Lilly \& Co., Indianapolis, IN, USA) given over $10 \mathrm{~min}$ and then an infusion at the rate of $80 \mathrm{mU} / \mathrm{m}^{2} / \mathrm{min}$. $20 \%$ glucose infusion was titrated to maintain arterialized blood glucose concentration at $100 \mathrm{mg} / \mathrm{dL}$. Insulin sensitivity was calculated from the glucose infusion rates during the last $30 \mathrm{~min}$ of $4-\mathrm{h}$ EHC (steady state) and expressed as glucose uptake per lean body mass (13). A biopsy from the vastus lateralis muscle was obtained at the start of the clamp, and then repeated on the contralateral vastus lateralis after $3.5 \mathrm{~h}$. The mean coefficient of variation of glucose during the clamps was $5.3 \%$. The steady state glucose and insulin concentrations were $99.7 \pm 5.3 \mathrm{mg} / \mathrm{dL}$ and $89 \mu \mathrm{U} / \mathrm{mL}$ (75-110 $\mathrm{uU} / \mathrm{mL})$ (13).

\section{Percutaneous skeletal muscle biopsy}

Muscle biopsies of vastus lateralis muscle were performed under local anesthesia. A $5 \mathrm{~mm}$ incision was made $10-15 \mathrm{~cm}$ above the patella and a biopsy needle connected to suction was inserted into the muscle. Approximately $200 \mathrm{mg}$ of muscle tissue was obtained, washed with PBS and then stored in liquid nitrogen $\left(-80^{\circ} \mathrm{C}\right)$ until processing for specific assays.

\section{DEXA scan}

Total and regional (appendicular and trunk) lean body mass and fat mass were measured by DEXA. The withincenter variability over time in measurement of lean mass is $0.9 \%$ and of fat mass is $1.2 \%(13)$.

\section{Quantification of mRNA expression by RT-PCR}

Expression of FGF2, FGFR1\&2, myogenin, MyoD, MRF4, MYF5 and myostatin were measured in skeletal muscle by RT-PCR. Total RNA was isolated from skeletal muscle using commercially available RNAqueous®-4PCR Kit (Ambion). 
Real-time RT-PCR was performed using Bio-Rad CFXConnect (Hercules, CA, USA), SYBR Green master mix (Qiagen) and gene-specific primers (Life Technologies). All values were normalized to the expression of a group of housekeeping genes including actin, ubiquitin $\mathrm{C}$ and cyclophilin A. The normalization factor used is calculated by GeneNorm software and is based on the values of all housekeeping genes used. There was not enough muscle tissue left to perform assessment of proteins of growth and differentiation genes.

FGF2 and plasma myostatin levels were measured by ELISA (R\&D Systems).

\section{Statistical analysis}

Group comparisons were performed by two-sided $t$ tests, Mann-Whitney rank-sum tests, and $\chi^{2}$ tests as appropriate. Percent change from baseline was calculated and statistical analysis for change from baseline was carried out using paired $t$-test or one-way repeatedmeasures ANOVA (RMANOVA) followed by Holm-Sidak post hoc test. Pearson correlation was performed between variables of interest to assess their relation to each other. All tests were performed using SPSS software (SPSS Inc). Data are presented as means \pm S.D. (or means \pm S.E. where indicated) for normally distributed data and median (25th, 75th percentile) for non-normal data. $P<0.05$ was considered significant.

Table 1 The baseline characteristics of study participants.

\begin{tabular}{|c|c|}
\hline & Values \\
\hline \multicolumn{2}{|l|}{ Parameter } \\
\hline Number of subjects & 20 \\
\hline Age (years) & $52 \pm 9$ \\
\hline $\mathrm{BMI}\left(\mathrm{kg} / \mathrm{m}^{2}\right)$ & $35 \pm 6$ \\
\hline Duration of diabetes (years) & $11 \pm 9$ \\
\hline HbA1c \% & $7.3 \pm 1.1$ \\
\hline Hemoglobin (g/dL) & $14.5 \pm 1.4$ \\
\hline Glucose infusion rate (mg/kg fat free mass/min) & $8.8 \pm 5.3$ \\
\hline \multicolumn{2}{|l|}{ Medications } \\
\hline Metformin & $80 \%$ \\
\hline Sulfonylureas & $30 \%$ \\
\hline Insulin & $40 \%$ \\
\hline GLP-1 agonists & $20 \%$ \\
\hline DPP-4 inhibitors & $15 \%$ \\
\hline Thiazolidinediones & $20 \%$ \\
\hline Diet only & $10 \%$ \\
\hline \multicolumn{2}{|l|}{ Ethnicity } \\
\hline Caucasian & $70 \%$ \\
\hline African American & $20 \%$ \\
\hline Hispanic & $10 \%$ \\
\hline
\end{tabular}

\section{Results}

Twenty men participated in the study. Demographics and clinical parameters of the study subjects are shown in Table 1 . These baseline characteristics are similar to the overall study population reported previously (13).

There was a significant increase in both myogenin (by $72 \pm 20 \%$ ) and MyoD (by $81 \pm 22 \%$ ) following EHC (Fig. 1). There was no change in MYF5 after EHC. The expression of MRF4 was suppressed significantly following EHC by $34 \pm 14 \%$ (Fig. 1). The expression of myostatin in skeletal muscle did not change following EHC. However, there was a decrease in plasma myostatin concentrations following EHC (from $3.4 \pm 0.4$ to $2.8 \pm 0.3 \mathrm{ng} / \mathrm{mL}, P=0.01$ ).

There was no change in expression of FGF2, but there was an increase in FGFR2 expression following EHC by $53 \pm 19 \%$. We did not find a change in FGFR1 (Fig. 1). There was no change in plasma concentrations of FGF2 following $\mathrm{EHC}$ (from $12.3 \pm 0.9$ to $11.8 \pm 1.2 \mathrm{pg} / \mathrm{mL}$, $P=0.82$ ).

Glucose infusion rate during EHC was not related to change in MyoD $(r=0.30, P=0.25)$, myogenin $(r=0.07$, $P=0.78)$, MRF4 $(r=-0.15, P=0.56)$ or FGFR2 $(r=0.42$, $P=0.11)$. Insulin concentrations during the clamp were not related to the change in FGFR2 $(r=0-0.02, P=0.93)$, MyoD $(r=0.31, P=0.42)$, myogenin $(r=0.41, P=0.29)$ and MRF4 $(r=0.62, P=0.10)$. The changes in FGFR2, MyoD, myogenin and MRF4 were similar in men who were on chronic insulin therapy versus those who were not

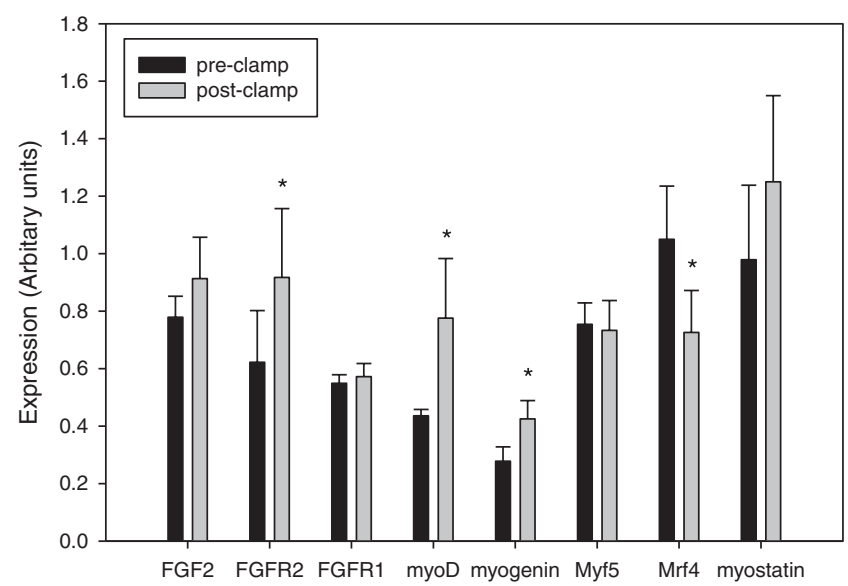

\section{Figure 1}

Effects of insulin on expression of FGF and muscle growth and differentiation genes (means \pm S.E.). $* P<0.05$ as compared to pre-clamp. The exact $P$ values are 0.26 (FGF2), 0.01 (FGFR2), 0.68 (FGFR1), 0.001 (MyoD), 0.001 (myogenin), 0.79 (MYF5), 0.03 (MRF4), 0.07 (myostatin). 
$(P=0.40,0.72,0.70$ and 0.79 respectively). The changes in FGFR2, MyoD, myogenin and MRF4 were similar in men who were on metformin versus those who were not $(P=0.50,0.71,0.39$ and 0.99 respectively). Hemoglobin A1c was not related to changes in FGFR2 $(r=0.10, P=0.68)$, MyoD $(r=-0.15, P=0.57)$, myogenin $(r=0.03, P=0.92)$ or MRF4 $(r=-0.35, P=0.16)$. Age was also not related to changes in FGFR2 $(r=0.14, P=0.58)$, MyoD $(r=0.12$, $P=0.64)$, myogenin $(r=0.21, P=0.44)$ or MRF4 $(r=-0.33$, $P=0.19)$.

\section{Conclusions}

The administration of insulin during the clamp procedure acutely led to a significant increase in the expression of two muscle growth factors, MyoD and myogenin. There was an increase in expression of FGFR2. FGF2 tended to increase, but it was not significant. Overall, these data suggest even $4 \mathrm{~h}$ of insulin administration can induce satellite cell activation and initiate muscle growth.

Insulin infusion led to a decrease in expression of muscle growth suppressor, MRF4. Plasma myostatin concentrations also decreased following EHC. Myostatin decreases satellite cell activation and diminishes myoblast maturation (14). However, EHC did not suppress the expression of myostatin in the muscle. The reason behind the discrepancy between serum myostatin and its expression in the muscle is not clear and needs further investigation.

There are limited studies on the effect of insulin on muscle growth. Most human studies have assessed muscle metabolism following insulin, glucose and amino acid infusion. Phenylalanine tracer studies consistently show that insulin infusion decreases muscle protein breakdown (4). Insulin also increases muscle protein synthesis, but only in the presence of enhanced amino acid delivery (4). An in vitro study in cultured myotubes has shown that insulin enhances expression of MyoD and myogenin (15). To our knowledge, our study is the first to report insulin's effects on myogenic regulatory factors in humans, in vivo. It is noteworthy that body-builders surreptitiously use insulin along with high protein and carbohydrate meals for muscle growth and performance enhancement $(16,17)$.

Our study has some limitations. (1) We evaluated the acute effects of insulin in the setting of EHC. It is not known if similar results would be obtained after chronic insulin treatment in patients with diabetes. (2) We did not fix muscle biopsy specimens for immunostaining or separate the satellite cells. Hence, we cannot directly observe the number or characteristics of satellite cells in our study. (3) We did not study intracellular signaling pathways of insulin's effect on myogenic transcription factors. Hence, we do not know if these effects are mediated via the insulin receptor or IGF receptor. (4) The insulin concentrations during our clamp studies are supra-physiological for lean, healthy subjects. However, they may approach the concentrations observed during the postprandial state in obesity and type 2 diabetes. We do not know if the gene expression results obtained from our experiments will be observed in clamps conducted with lower insulin doses. (5) The study was conducted in men. We do not know if the results are applicable to women. (6) This is an observational study and we do not have a control group that did not have EHC performed. (7) We did not collect data on exercise regimens of the study participants. However, none of the patients were following a planned exercise routine at the time of recruitment.

In conclusion, our data show that an acute insulin infusion increases FGFR2, MyoD and myogenin expression in skeletal muscle, while suppressing MRF4. These effects support a role for insulin in modulating anabolic actions in skeletal muscle.

\section{Declaration of interest}

S D: Bayer (consultant). A C: Eli Lilly, Sanofi-Aventis (speaker panel). P D: Research Support: National Institutes of Health; JDRF, ADA; Novo Nordisk; Bristol Meyer Squibb; AbbVie Pharmaceuticals; Astra Zeneca, Boehringer Ingelheim Pharmaceuticals. Honorarium: Eli Lilly; Novartis; GlaxoSmithKline; Merck; Novo Nordisk; Takeda; Sanofi-Aventis. The other authors have nothing to disclose.

\section{Funding}

Supported by National Institute of Diabetes and Digestive and Kidney Diseases (NIDDK) (R01 grant to P D; R01DK075877).

\section{Author contribution statement}

P D put forth the hypothesis, planned and interpreted the study, and wrote the manuscript. $H$ G and $S D$ analyzed data and wrote the manuscript. H G, S A and K G analyzed samples. S D, M B, S S, A C and A M executed the study. P D, S D and H G are the guarantors of this work and, as such, have full access to all the data in the study and take responsibility for the integrity of the data and the accuracy of the data analysis.

\section{Acknowledgement}

The authors are grateful to Zahid Sayeed for assistance in formatting and submission of this manuscript. 


\section{References}

1 Mandel JL \& Pearson ML. Insulin stimulates myogenesis in a rat myoblast line. Nature 1974251 618-620. (https://doi. org/10.1038/251618a0)

2 Park SW, Goodpaster BH, Strotmeyer ES, de Rekeneire N, Harris TB, Schwartz AV, Tylavsky FA \& Newman AB. Decreased muscle strength and quality in older adults with type 2 diabetes: the health, aging, and body composition study. Diabetes 200655 1813-1818. (https:// doi.org/10.2337/db05-1183)

3 Park SW, Goodpaster BH, Strotmeyer ES, Kuller LH, Broudeau R, Kammerer C, de Rekeneire N, Harris TB, Schwartz AV, Tylavsky FA et al. Accelerated loss of skeletal muscle strength in older adults with type 2 diabetes: the health, aging, and body composition study. Diabetes Care 200730 1507-1512. (https://doi.org/10.2337/dc06-2537)

4 Abdulla H, Smith K, Atherton PJ \& Idris I. Role of insulin in the regulation of human skeletal muscle protein synthesis and breakdown: a systematic review and meta-analysis. Diabetologia 2016 59 44-55. (https://doi.org/10.1007/s00125-015-3751-0)

5 Zanou N \& Gailly P. Skeletal muscle hypertrophy and regeneration: interplay between the myogenic regulatory factors (MRFs) and insulinlike growth factors (IGFs) pathways. Cellular and Molecular Life Sciences 201370 4117-4130. (https://doi.org/10.1007/s00018-013-1330-4)

6 Pawlikowski B, Vogler TO, Gadek K \& Olwin BB. Regulation of skeletal muscle stem cells by fibroblast growth factors. Developmental Dynamics 2017246 359-367. (https://doi.org/10.1002/dvdy.24495)

7 O'Connor RS \& Pavlath GK. Point:Counterpoint: satellite cell addition is/is not obligatory for skeletal muscle hypertrophy. Journal of Applied Physiology 2007103 1099-1100. (https://doi.org/10.1152/ japplphysiol.00101.2007)

8 Almada AE \& Wagers AJ. Molecular circuitry of stem cell fate in skeletal muscle regeneration, ageing and disease. Nature Reviews: Molecular Cell Biology 201617 267-279. (https://doi.org/10.1038/nrm.2016.7)

9 Egner IM, Bruusgaard JC \& Gundersen K. Satellite cell depletion prevents fiber hypertrophy in skeletal muscle. Development 2016143 2898-2906. (https://doi.org/10.1242/dev.134411)
10 Moretti I, Ciciliot S, Dyar KA, Abraham R, Murgia M, Agatea L, Akimoto T, Bicciato S, Forcato M, Pierre P et al. MRF4 negatively regulates adult skeletal muscle growth by repressing MEF2 activity. Nature Communications 20167 12397. (https://doi.org/10.1038/ ncomms12397)

11 Zammit PS. Function of the myogenic regulatory factors Myf5, MyoD, myogenin and MRF4 in skeletal muscle, satellite cells and regenerative myogenesis. Seminars in Cell and Developmental Biology 201772 19-32. (https://doi.org/10.1016/j.semcdb.2017.11.011)

12 Ghanim H, Dhindsa S, Batra M, Green K, Abuaysheh S, Kuhadiya ND, Makdissi A, Chaudhuri A \& Dandona P. Effect of testosterone on FGF2, MRF4 and myostatin in hypogonadotropic hypogonadism: relevance to muscle growth. Journal of Clinical Endocrinology and Metabolism 2019104 2094-2102. (https://doi. org/10.1210/jc.2018-01832)

13 Dhindsa S, Ghanim H, Batra M, Kuhadiya ND, Abuaysheh S, Sandhu S, Green K, Makdissi A, Hejna J, Chaudhuri A et al. Insulin resistance and inflammation in hypogonadotropic hypogonadism and their reduction after testosterone replacement in men with type 2 diabetes. Diabetes Care 201639 82-91. (https://doi.org/10.2337/ dc15-1518)

14 Sharma M, McFarlane C, Kambadur R, Kukreti H, Bonala S \& Srinivasan S. Myostatin: expanding horizons. IUBMB Life 201567 589-600. (https://doi.org/10.1002/iub.1392)

15 Litwiniuk A, Pijet B, Pijet-Kucicka M, Gajewska M, Pajak B \& Orzechowski A. FOXO1 and GSK-3beta are main targets of insulinmediated myogenesis in C2C12 muscle cells. PLoS ONE 201611 e0146726. (https://doi.org/10.1371/journal.pone.0146726)

16 Pope Jr HG, Wood RI, Rogol A, Nyberg F, Bowers L \& Bhasin S. Adverse health consequences of performance-enhancing drugs: an Endocrine Society scientific statement. Endocrine Reviews 201435 341-375. (https://doi.org/10.1210/er.2013-1058)

17 Ip EJ, Barnett MJ, Tenerowicz MJ \& Perry PJ. Weightlifting's risky new trend: a case series of 41 insulin users. Current Sports Medicine Reports 201211 176-179. (https://doi.org/10.1249/ JSR.0b013e31825da97f)

Received 5 July 2019

Revised version received 8 October 2019

Accepted 15 October 2019 